\title{
Ideality of a Coisotropic Lightlike Submanifold
}

\author{
Erol Kılıç and Mehmet Gülbahar*
}

(Communicated by Krishan Lal DUGGAL)

\begin{abstract}
The notion of best living way on coisotropic lightlike submanifolds is discussed. Some relations involving the screen Ricci curvature and the screen scalar curvature are given. Two examples of coisotropic lightlike submanifolds are mentioned and ideals of leaves of screen distributions in these examples are investigated by the help of these relations.
\end{abstract}

Keywords: Ideal submanifold; lightlike submanifold; curvature.

AMS Subject Classification (2010): Primary: 46C20; Secondary: 53C40; 53C42.

\section{Introduction}

In 1998, B.-Y. Chen [3] firstly introduced ideal immersions in Riemannian context. He called a Riemannian submanifold in a space form as an ideal submanifold if it receives the least possible amount of tension from the surrounding space. Also, he explained physical interpretation of ideal immersions as follows:

A submanifold $M$ is an ideal submanifold means that $M$ lives in the best world in a best way. The best world is a space which has the highest degree of homogeneity. Euclidean spaces, spheres, real projective spaces, real hyperbolic spaces are mainly examples of the best worlds. In this sense, a best world in differential geometry could be considered as a Riemannian space form $R^{m}(c)$ of constant curvature $c$. Furthermore, if a space is affected as little as possible from the external influence, then we say that it has best way of living. Thus, every ideal immersion $x: M \rightarrow R^{m}(c)$ indicates a best living way in the best world.

In this direction, B.-Y. Chen established the following important inequality for an $n$-dimensional Riemannian submanifold $M$ of an Euclidean space in [3]:

$$
\|H\|^{2} \geq \frac{2\left(n+k-\sum_{j=1}^{k} n_{j}\right)}{n^{2}\left(n+k-1-\sum_{j=1}^{k} n_{j}\right)} \delta\left(n_{1}, \ldots, n_{k}\right),
$$

where $H$ is the mean curvature vector of $M$ and

$$
\delta\left(n_{1}, \ldots, n_{k}\right)=\tau(p)-\inf \left\{\tau\left(\pi_{n_{1}}\right)+\cdots+\tau\left(\pi_{n_{k}}\right)\right\}
$$

for $\pi_{n_{1}}, \ldots, \pi_{n_{k}}$ run over all $k$ mutually orthogonal subspaces of $T_{p} M$ with $\operatorname{dim} \pi_{j}=n_{j}, j=1, \ldots, k$. Since the squared mean curvature measures the amount of the tension at $p \in M$, the equality case of (1.1) holds for all $p \in M$ if and only if $M$ is an ideal submanifold.

Later, ideal submanifolds have been attracted by many geometers. This topic was studied on submanifolds of real space forms in $[4,9,10,17,26,27]$ etc., of complex space forms in $[5,8,20,25,31,32,35,36]$ etc., of contact space forms in $[7,24,34]$ etc., of nearly constant curvature manifolds [28, 29, 37, 38] etc. Recently, B.-Y. Chen [6] published a book related with applications of $\delta$-curvatures and ideal submanifolds in semi-Riemannian manifolds. 
Considering the above facts, it shall be interesting to investigate the notion of ideality in lightlike (degenerate) submanifolds. But, since any lightlike submanifold contains a radical space and thus trace of the second fundamental form is meaningless, it doesn't enable to study the notion of best living way on a lightlike submanifold. For this reason, we shall investigate to ideality on any leaf of screen distribution of screen conformal coisotropic lightlike submanifolds in a semi-Euclidean space of index 2 in this paper.

\section{Preliminaries}

In this section, we recall some basic facts about coisotropic lightlike submanifolds by following the notations and formulas used in $[12,13,16,21,22]$.

An $(n+2)$-dimensional $(n \geq 1)$ lightlike submanifold $(M, g, S(T M))$ of a semi-Riemannian manifold $(\widetilde{M}, \widetilde{g})$ of codimension 2 is called a coisotropic lightlike submanifold if it is a submanifold with the degenerate metric $g$ induced from $\widetilde{g}$ and the rank of radical distribution $\operatorname{Rad} T M$ is of 2 . In this case, $\operatorname{Rad} T M=T M^{\perp}$. Here, $S(T M)$ denotes an $n$-dimensional complementary Riemannian distribution of $\operatorname{Rad} T M$, that is

$$
T M=\operatorname{Rad} T M \oplus_{\text {orth }} S(T M),
$$

where $\oplus_{\text {orth }}$ denotes the orthogonal direct sum. From (2.1) there exists a field of frame

$$
\left\{\xi_{1}, \xi_{2}, e_{1}, \ldots, e_{n}\right\}
$$

on a coordinate neigborhood $\mathcal{U}$ on $M$, where $\left.\operatorname{Rad} T M\right|_{\mathcal{U}}=\operatorname{Span}\left\{\xi_{1}, \xi_{2}\right\}$ and $\left.S(T M)\right|_{\mathcal{U}}=\operatorname{Span}\left\{e_{1}, \ldots e_{n}\right\}$. For any basis $\left\{\xi_{i}\right\}$ of $\left.\operatorname{Rad} T M\right|_{\mathcal{U}}$ it is known that there exist smooth sections $\left\{N_{i}\right\}$ of $\left.S\left(T M^{\perp}\right)\right|_{\mathcal{U}}$ satisfying

$$
\widetilde{g}\left(N_{i}, \xi_{j}\right)=\delta_{i j}, \quad \widetilde{g}\left(N_{i}, N_{j}\right)=0
$$

for $i, j=1,2$.

The complementary vector bundle of $\operatorname{Rad} T M$ in $S\left(T M^{\perp}\right)$, denoted by $\operatorname{tr}(T M)$, spanned by the vectors $N_{1}$ and $N_{2}$, is called transversal bundle of $M$. Thus, we have

$$
T \widetilde{M}=S(T M) \oplus_{\text {orth }}(\operatorname{Rad} T M \oplus \operatorname{tr}(T M)),
$$

where $\oplus$ denote the diret sum, but it is not orthogonal.

Let $\widetilde{\nabla}$ be the Levi-Civita connection on $\widetilde{M}$. The Gauss and Weingarten formulas are given by

$$
\begin{aligned}
& \widetilde{\nabla}_{X} Y=\nabla_{X} Y+h(X, Y), \\
& \widetilde{\nabla}_{X} N_{k}=-A_{N_{k}} X+\nabla_{X}^{t} N_{k}
\end{aligned}
$$

for all $X, Y \in \Gamma(T M)$, where $\nabla_{X} Y, A_{N_{k}} X \in \Gamma(T M)$ and $h(X, Y), \nabla_{X}^{t} N_{k} \in \Gamma(\operatorname{tr}(T M))$. Here, $h$ is called second fundamental form and $A_{N_{k}}$ are called shape operators of $M$. If we put

$$
D^{k}(X, Y)=\widetilde{g}\left(h(X, Y), \xi_{k}\right) \text { and } \rho_{k \ell}(X)=\widetilde{g}\left(\nabla_{X}^{t} N_{k}, \xi_{\ell}\right)
$$

for $k, \ell=1,2$, then the equations (2.5) and (2.6) become

$$
\begin{aligned}
& \widetilde{\nabla}_{X} Y=\nabla_{X} Y+\sum_{k=1}^{2} D^{k}(X, Y) N_{k}, \\
& \widetilde{\nabla}_{X} N_{k}=-A_{N_{k}} X+\sum_{\ell=1}^{2} \rho_{k \ell}(X) N_{\ell},
\end{aligned}
$$

respectively. Consider (2.3) and (2.7), we obtain $D^{k}$ are symmetric, independent of choosing screen distribution and satisfy

$$
D^{k}\left(X, \xi_{k}\right)=0 \text { and } D^{k}\left(X, \xi_{\ell}\right)=-D^{\ell}\left(X, \xi_{k}\right)
$$

for all $X \in \Gamma(T M)$ and $k \neq \ell=1,2$. 
Let us denote the projection of $T M$ to $S(T M)$ by $P$. From (2.1) we get

$$
\begin{aligned}
\nabla_{X} P Y & =\nabla_{X}^{*} P Y+h^{*}(X, Y) \\
& =\nabla_{X}^{*} P Y+\sum_{k=1}^{2} C^{k}(X, P Y) \xi_{k}, \\
\nabla_{X} \xi_{k} & =-A_{\xi_{k}}^{*} X-\sum_{\ell=1}^{2} \rho_{k \ell}(X) \xi_{\ell} .
\end{aligned}
$$

Here, $h^{*}$ is called local second fundamental form and $A_{\xi_{k}}^{*}$ are called local shape operators on $S(T M)$. Using (2.3), (2.5), (2.7), (2.10) and (2.11), we also have the following equations:

$$
D^{k}(X, Y)=g\left(A_{\xi_{k}}^{*} X, Y\right), \quad C^{k}(X, P Y)=g\left(A_{N_{k}} X, P Y\right)
$$

for all $k \in\{1,2\}$.

The submanifold $(M, g, S(T M))$ is called irrotational [23] if $D^{k}$ vanish on Rad $T M$ and it is called totally geodesic [13] if $D^{k}$ vanish identically on $\Gamma(T M)$ for all $k=1,2$. If there exist smooth functions $\lambda_{k}^{\prime}$ on $\operatorname{tr}(T M)$ satisfying

$$
D^{k}(X, Y)=\lambda_{k}^{\prime} \widetilde{g}(X, Y)
$$

for all $X, Y \in \Gamma(T M)$, then the submanifold is called totally umbilical [14].

Let $\left\{e_{1}, \ldots, e_{n}\right\}$ be an orthonormal basis of $\Gamma(S(T M))$. Define

$$
\mu_{1}=\frac{1}{n} \sum_{j=1}^{n} D^{1}\left(e_{j}, e_{j}\right) \text { and } \mu_{2}=\frac{1}{n} \sum_{j=1}^{n} D^{2}\left(e_{j}, e_{j}\right) .
$$

The submanifold is called minimal if $\mu_{1}=\mu_{2}=0$ [1].

Let $\varphi_{k}$ be non-zero functions on a neigborhood $\mathcal{U}$. The submanifold is called screen locally conformal if the shape operators $A_{N_{k}}$ and $A_{\xi_{k}}^{*}$ of $M$ and $S(T M)$, respectively, are related by

$$
A_{N_{k}}=\varphi_{k} A_{\xi_{k}}^{*},
$$

i.e.

$$
C^{k}(X, P Y)=\varphi_{k} D^{k}(X, Y)
$$

for all $X, Y \in \Gamma(T M)$.

Denote curvature tensors of the ambient manifold and the submanifold by $\widetilde{R}$ and $R$, respectively. Then the following relation between these tensors holds:

$$
\begin{aligned}
& \widetilde{g}(\widetilde{R}(X, Y) P Z, P W)=g(R(X, Y) P Z, P W) \\
& +\sum_{k=1}^{2}\left[D^{k}(X, P Z) C^{k}(Y, P W)-D^{k}(Y, P Z) C^{k}(X, P W)\right]
\end{aligned}
$$

for all $X, Y, Z, W \in \Gamma(T M)[21]$.

Let $\Pi=\operatorname{Span}\left\{e_{i}, e_{j}\right\}$ be a 2-dimensional non-degenerate plane in $T_{p} M$. Then the sectional curvature at $p$ is expressed by

$$
K_{i j}=\frac{g\left(R\left(e_{i}, e_{j}\right) e_{j}, e_{i}\right)}{g\left(e_{i}, e_{i}\right) g\left(e_{j}, e_{j}\right)-g\left(e_{i}, e_{j}\right)^{2}}
$$

We note that since $C^{k}$ aren't symmetric, it is clear from (2.17) that the sectional curvature map does not need to be symmetric for any lighlike submanifold of a semi-Riemannian manifold.

Now, we recall the following result [13]: 
Theorem 2.1. Let $(M, g, S(T M))$ be an r-lightlike submanifold of a semi-Riemannian manifold $(\widetilde{M}, \widetilde{g})$. Then the following assertions are equivalent:

i) $S(T M)$ is integrable.

ii) $h^{*}$ is symmetric on $\Gamma(S(T M))$.

iii) $A_{N}$ is self-adjoint on $\Gamma(S(T M))$ with respect to $g$.

As a consequence of Theorem 2.1, we see that the sectional curvature is symmetric on every screen conformal lightlike submanifold of a semi-Riemannian manifold.

\section{Ideality of lightlike submanifolds}

In this section, we start by recalling some basic facts about the energy equation and harmonicity in Riemannian geometry and semi-Riemannian geometry.

Let $\sigma:(M, g) \rightarrow(\widetilde{M}, \widetilde{g})$ be a smooth map between Riemannian manifolds $(M, g)$ and $(\widetilde{M}, \widetilde{g})$. The energy density at a point $p \in M$, denoted by $e(\sigma)$, is defined by

$$
e(\sigma)=\frac{1}{2}\|d \sigma\|^{2}
$$

where $\|d \sigma\|^{2}$ is the Hilbert-Schmidt norm of the differential of $\sigma$, given by $\|d \sigma\|^{2}=\operatorname{trace}_{g} \sigma^{*}(\widetilde{g})$, with respect to the induced metric on the bundle $T^{*} M \otimes \sigma^{-1}(T \widetilde{M})$. The total energy of $\sigma$ is given by

$$
E(\sigma)=\int_{\mathcal{D}} e(\sigma) d v_{g},
$$

where $d v_{g}$ denotes the measure on $M$ induced by its metric and $\mathcal{D}$ denotes any compact domain in $M$.

The mapping $\sigma$ is called harmonic if it is a critical point of the energy functional $E$, that is

$$
\tau(\sigma)=\operatorname{trace}_{g}\left(\nabla^{\sigma} d \sigma\right)=0,
$$

where $\nabla^{\sigma}$ is the connection on the vector bundle $T^{*} M \otimes \sigma^{-1}(T \widetilde{M})$ induced by the Levi-Civita connections on $M$ and $\widetilde{M}$. Here, $\tau(\sigma)$ is a section of the bundle $\sigma^{-1}(T \widetilde{M})$, called tension field of $\sigma$. Also, it is known that any harmonic map is a $C^{2}$-smooth local solution of the Laplace-Beltrami equation, given in local coordinates $x^{i}$ by

$$
\Delta_{M}=\frac{1}{\sqrt{\left|\operatorname{det}\left(g_{i j}\right)\right|}} \sum_{i} \partial x^{i}\left(\sum_{i} g^{i j} \partial x_{j}\right),
$$

where $g^{i j}$ is the inverse of the metric $g$.

Hovewer, in semi-Riemannian context, integration of a compact domain is questionable since the HopfRinow theorem doesn't hold for a semi-Riemannian manifold and due to the possibility of degenerate fibers in this domain. Thus, the harmonic maps between semi-Riemannian manifolds or lightlike manifolds behave differently from the Riemannian context.

Now, let $\sigma:(M, g) \rightarrow(\widetilde{M}, \widetilde{g})$ be a smooth map. Let us consider the following two cases:

Case 1: $M$ is a Riemannian or semi-Riemannian manifold and $\widetilde{M}$ is lightlike manifold.

Case 2: $M$ is a lightlike manifold and $\widetilde{M}$ is a semi-Riemannian manifold.

For the case 1 , the tension field can not be defined since $\widetilde{g}$ is degenerate and thus $\sigma^{-1}(T \widetilde{M})$ will not exist. In this direction, K. L. Duggal [11] introduced a special class of lightlike submanifolds in which harmonic maps can be defined for the first case as follows:

Definition 3.1. A lightlike manifold is called globally null if it admits a globally null vector field and a complete Riemannian hypersurface. 
Let $(M, g)$ be a Riemannian (or semi-Riemannian) manifold and $(\widetilde{M}, \widetilde{g})$ be a globally null manifold. Consider a smooth $\operatorname{map} \sigma:(M, g) \rightarrow(\widetilde{M}, \widetilde{g})$ defined by

$$
\sigma: M \rightarrow \widetilde{M^{\prime}} \subset \widetilde{M}, \sigma(M)=\widetilde{M^{\prime}},
$$

where $\widetilde{M}$ denotes a complete Riemannian hypersurface of $\widetilde{M}$. Then the pullback $\sigma^{-1}\left(T \widetilde{M}^{\prime}\right)$ exists and thus, $\sigma$ is a harmonic map if and only if the equation (3.4) holds or $\tau(\sigma)=0$.

For the case 2, harmonic maps also can not be defined since trace of the second fundamental form is meaningless on the radical space. To heal this, B. Sahin stated the following definition in [30].

Definition 3.2. Let $(M, g)$ be a lightlike manifold and $(\widetilde{M}, \widetilde{g})$ be a semi-Riemannian manifold. A smooth map $\sigma: M \rightarrow \widetilde{M}$ is called harmonic if the following assertions hold:

i) $\nabla d \sigma=0$ on $\operatorname{Rad} T M$.

ii) trace $\left.\right|_{S(T M)} \nabla d \sigma=0$, where the trace $\left.\right|_{S(T M)}$ denotes the trace restricted to $S(T M)$ with respect to the degenerate metric $g$.

It is clear from Definition 3.2 that if $\sigma: M \rightarrow \widetilde{M}$ is an $r$-lightlike immersion, then $d \sigma$ is equal to the second fundamental form given in (2.5). Thus, an $r$-lightlike immersion is harmonic if and only if it is a minimal immersion.

From the above facts we state the following definition:

Definition 3.3. Let $(M, g, S(T M))$ be a lightlike submanifold of an $(n+m)$-dimensional semi-Euclidean space $R_{q}^{n+m}$. Suppose $S(T M)$ is integrable and $\left(M^{\prime}, g^{\prime}\right)$ is an $n$-dimensional leaf of $S(T M)$ immersed in $R_{q}^{n+m}$ as a co-dimension $m$ with the non-degenerate metric $g^{\prime}$. The leaf $\left(M^{\prime}, g^{\prime}\right)$ is called ideal if $\left\|H^{\prime}\right\|^{2}$ the Hilbert-Schmidt norm of the mean curvature vector $H^{\prime}(p)$ of $M^{\prime}$ takes optimum value at every point of it.

Now, consider $(M, g, S(T M))$ is an $(n+2)$-dimensional coisotropic lightlike submanifold of an $(n+4)$ dimensional semi-Euclidean space $R_{2}^{n+4}$ and $S(T M)$ is an integrable distribution. If $M^{\prime}$ is an $n$-dimensional leaf on $S(T M)$, from (2.6) and (2.10) we have

$$
\nabla_{X} Y=\nabla_{X}^{\prime} Y+\sum_{k=1}^{2} C^{k}(X, Y) \xi_{k}+\sum_{k=1}^{2} D^{k}(X, Y) N_{k}
$$

for all $X, Y \in \Gamma\left(T M^{\prime}\right)$, where $\nabla^{\prime}$ is the induced connection of $M^{\prime}$. Here, the second fundamental form $h^{\prime}$ of $M^{\prime}$ is given by

$$
h^{\prime}(X, Y)=\sum_{k=1}^{2} C^{k}(X, Y) \xi_{k}+\sum_{k=1}^{2} D^{k}(X, Y) N_{k}
$$

and the mean curvature vector $H^{\prime}(p)$ of $M^{\prime}$ satisfies

$$
\begin{aligned}
H^{\prime}(p) & =\left.\operatorname{trace}\right|_{S(T M)}\left(h^{\prime}\right) \\
& =\sum_{i=1}^{n} \sum_{k=1}^{2} C^{k}\left(e_{i}, e_{i}\right) \xi_{k}+\sum_{k=1}^{2} \mu_{k} N_{k},
\end{aligned}
$$

where $\left\{e_{1}, \ldots, e_{n}\right\}$ is an orthonormal basis of $\Gamma(S(T M))$.

From (2.16) and (3.7) if $(M, g, S(T M))$ is screen conformal, then it follows that

$$
n H^{\prime}(p)=\sum_{k=1}^{2}\left[\mu_{k} N_{k}+\varphi_{k} \mu_{k} \xi_{k}\right]
$$

and

$$
n^{2}\left\|H^{\prime}(p)\right\|^{2}=2 \sum_{k=1}^{2} \varphi_{k} \mu_{k}^{2}
$$

where $\mu_{k}$ is given in (2.14). 


\section{Some relations for coisotropic lightlike submanifolds}

We begin this section with the following definition:

Definition 4.1. Let $(M, g, S(T M))$ be an $(n+2)$-dimensional screen conformal lightlike submanifold of an $(n+4)$-dimensional semi-Riemannian manifold of index 2 . The screen Ricci curvature tensor, denoted by $\operatorname{Ric}_{S(T M)}$, is defined by

$$
\operatorname{Ric}_{S(T M)}(X, Y)=\operatorname{trace}_{S(T M)}\{Z \rightarrow R(X, Z) Y\}
$$

for any $X, Y \in \Gamma(S(T M))$.

Suppose that $\left\{e_{1}, \ldots, e_{n}\right\}$ be an orthonormal basis of $\Gamma(S(T M))$. The screen Ricci curvature at a unit vector $e_{i} \in \Gamma(S(T M))$, denoted by $\operatorname{Ric}_{S(T M)}\left(e_{i}\right)$, is given by

$$
\operatorname{Ric}_{S(T M)}\left(e_{i}\right)=\sum_{j \neq i=1}^{n} g\left(R\left(e_{i}, e_{j}, e_{j}\right) e_{i}\right)=\sum_{j \neq i=1}^{n} K_{i j}
$$

We note that since the screen distribution is Riemannian, it is clear that the sectional curvature map can be bounded and so the screen Ricci curvature can also be bounded. This features enable to studying some comparision theorems for coisotropic lightlike submanifolds. Also,

a) If $n=1$, the screen Ricci curvature vanishes identically.

b) If $n=2$, the screen Ricci curvature is equal to the sectional curvature.

Remark 4.1. The screen Ricci curvature was firstly introduced by K. L. Duggal in [15] and named by the authors in $[18,19]$ for a lightlike hypersurface of a Lorentzian manifold.

Definition 4.2. Let $(M, g, S(T M))$ be an $(n+2)$-dimensional screen conformal lightlike submanifold of an $(n+4)$-dimensional semi-Riemannian manifold of index 2. Suppose $\left\{e_{1}, \ldots, e_{n}\right\}$ is an orthonormal basis of $\Gamma(S(T M))$. The screen scalar curvature at a point $p \in M$, denoted by $r_{S(T M)}(p)$, is defined by

$$
r_{S(T M)}(p)=\sum_{i, j=1}^{n} K_{i j}
$$

Taking into consideration the equations (2.16), (2.17) and (4.3), we have

$$
r_{S(T M)}(p)=\widetilde{r}_{S(T M)}(p)+\sum_{k=1}^{2} \varphi_{k} \sum_{i, j=1}^{n} D_{i i}^{k} D_{j j}^{k}-\left(D_{i j}^{k}\right)^{2} .
$$

where $D_{i j}^{k}=D^{k}\left(e_{i}, e_{j}\right)$ for $i, j \in\{1, \ldots, n\}, k \in\{1,2\}$ and $\widetilde{r}_{T_{p} M}(p)$ is the scalar curvature of screen distribution of $\widetilde{M}$ (see the equation (2.3) in [20]) given by

$$
\widetilde{r}_{S(T M)}(p)=\sum_{i, j=1}^{n} \widetilde{g}\left(\widetilde{R}\left(e_{i}, e_{j}\right) e_{j}, e_{i}\right) .
$$

Theorem 4.1. Let $(M, g, S(T M))$ be an $(n+2)$-dimensional screen conformal coisotropic lightlike submanifold of an $(n+4)$-dimensional semi-Riemannian manifold $(\widetilde{M}, \widetilde{g})$. Then we have the following statements:

a) If $\varphi_{k}>0$ for all $k=1,2$, then

$$
2 r_{S(T M)}(p) \leq 2 \widetilde{r}_{S(T M)}(p)+n^{2}\left\|H^{\prime}(p)\right\|^{2} .
$$

The equality case of (4.6) holds for all $p \in M$ if and only if $S(T M)$ is totally geodesic.

b) If $\varphi_{k}<0$ for all $k=1,2$, then

$$
2 r_{S(T M)}(p) \geq 2 \widetilde{r}_{S(T M)}(p)+n^{2}\left\|H^{\prime}(p)\right\|^{2} .
$$

The equality case of (4.7) holds for all $p \in M$ if and only if $S(T M)$ is totally geodesic. 
c) If $\varphi_{1}>0$ and $\varphi_{2}<0$, then

$$
2 r_{S(T M)}(p) \leq 2 \widetilde{r}_{S(T M)}(p)+n^{2}\left\|H^{\prime}(p)\right\|^{2}-2 \sum_{i, j=1}^{n} \varphi_{2}\left(D_{i j}^{2}\right)^{2} .
$$

The equality case of (4.8) holds for all $p \in M$ if and only if $D^{1}$ vanishes on $S(T M)$.

d) If $\varphi_{1}<0$ and $\varphi_{2}>0$, then

$$
2 r_{S(T M)}(p) \leq 2 \widetilde{r}_{S(T M)}(p)+n^{2}\left\|H^{\prime}(p)\right\|^{2}-2 \sum_{i, j=1}^{n} \varphi_{1}\left(D_{i j}^{1}\right)^{2} .
$$

The equality case of (4.9) holds for all $p \in M$ if and only if $D^{2}$ vanishes on $S(T M)$.

Proof. If we put (3.9) in the equation (4.4), we have

$$
2 r_{S(T M)}(p)=2 \widetilde{r}_{S(T M)}(p)+n^{2}\left\|H^{\prime}(p)\right\|^{2}-2 \sum_{k=1}^{2} \varphi_{k} \sum_{i, j=1}^{n}\left(D_{i j}^{k}\right)^{2} .
$$

The rest part proof of theorem is straightforward.

From Theorem 4.1 we get the following proposition:

Proposition 4.1. Let $(M, g, S(T M))$ be an $(n+2)$-dimensional screen conformal coisotropic lightlike submanifold of an $(n+4)$-dimensional semi-Euclidean space $R_{2}^{n+4}$.

a) If $\varphi_{k}>0$ for all $k=1,2$ and $r_{S(T M)}>0$, then any $n$-dimensional leaf of $S(T M)$ does not admit a minimal immersion.

b) If $\varphi_{k}<0$ for all $k=1,2$ and $r_{S(T M)}<0$, then any $n$-dimensional leaf of $S(T M)$ does not admit a minimal immersion.

As a consequence of Proposition 4.1, it is clear that any harmonic (or minimal) map is ideal but the converse part is not always true.

Now, we shall need to following lemma for later uses:

Lemma 4.1. [33] If $a_{1}, \ldots, a_{n}$ are $n$-real numbers $(n>1)$, then

$$
\frac{1}{n}\left(\sum_{i=1}^{n} a_{i}\right)^{2} \leq \sum_{i=1}^{n} a_{i}^{2}
$$

with equality if and only if $a_{1}=\ldots=a_{n}$.

Theorem 4.2. Let $(M, g, S(T M))$ be an $(n+2)$-dimensional screen conformal coisotropic lightlike submanifold of an $(n+4)$-dimensional semi-Riemannian manifold $(\widetilde{M}, \widetilde{g})$. Then we have the following statements:

a) If $\varphi_{k}>0$ for all $k=1,2$, then

$$
2 r_{S(T M)}(p) \leq 2 \widetilde{r}_{S(T M)}(p)+n(n-1)\left\|H^{\prime}(p)\right\|^{2} .
$$

The equality case of (4.12) holds for all $p \in M$ if and only if $M$ is totally umbilical.

b) If $\varphi_{k}<0$ for all $k=1,2$, then

$$
2 r_{S(T M)}(p) \leq 2 \widetilde{r}_{S(T M)}(p)+n(n-1)\left\|H^{\prime}(p)\right\|^{2}-2 \sum_{k=1}^{2} \varphi_{k} \sum_{i \neq j=1}^{n}\left(D_{i j}^{k}\right)^{2} .
$$

The equality case of (4.13) holds for all $p \in M$ if and only if $\mu_{k}=D^{k}(X, X)$ for all unit vector $X \in \Gamma(S(T M))$ and $k=1,2$.

c) If $\varphi_{1}>0$ and $\varphi_{2}<0$, then

$$
2 r_{S(T M)}(p) \leq 2 \widetilde{r}_{S(T M)}(p)+n(n-1)\left\|H^{\prime}(p)\right\|^{2}-2 \varphi_{2} \sum_{i \neq j=1}^{n}\left(D_{i j}^{2}\right)^{2} .
$$


The equality case of (4.14) holds for all $p \in M$ if and only if

$$
D^{1}(X, Y)=\lambda_{1} g(X, Y), \text { for all } X, Y \in \Gamma(S(T M)),
$$

where $\lambda_{1}$ is a smooth function on $\operatorname{ltr}(T M)$.

Proof. Using (4.4), we have

$$
\begin{aligned}
r_{S(T M)}(p)= & \widetilde{r}_{S(T M)}(p)+n^{2} \sum_{k=1}^{2} \varphi_{k} \mu_{k}^{2}-\sum_{k=1}^{2} \varphi_{k} \sum_{i \neq j=1}^{n}\left(D_{i j}^{k}\right)^{2} \\
& -\sum_{k=1}^{2} \varphi_{k} \sum_{i=1}^{n}\left(D_{i i}^{k}\right)^{2} .
\end{aligned}
$$

If we take $\varphi_{k}>0$ for all $k \in\{1,2\}$ and use Lemma 4.1 in (4.16), we get (4.14).

The equality case of (4.14) holds for all $p \in M$ if and only if

$$
D_{11}^{k}=\cdots=D_{n n}^{k}, D_{i j}=0, \text { for all } i \neq j \in\{1, \ldots, n\} \text { and } k \in\{1,2\},
$$

which shows that $M$ is totally umbilical with $\lambda_{k}=1$. This is the proof of the $(a)$ statement of the theorem.

Similarly, one can prove the statements (b) and (c).

Now, we recall the following theorem of D. H. Jin in [21].

Theorem 4.3. Let $(M, g, S(T M))$ be a proper totally umbilical coisotropic submanifold of semi-Riemannian space form $\widetilde{M}(c)$ of constant curvature $c$. If the screen distribution $S(T M)$ is totally geodesic, then $c=0$.

From (a) statements (or (b) statements) of both Theorem 4.1 and Theorem 4.2 we get the following corollary immediately:

Corollary 4.1. Let $M$ be an $(n+2)$-dimensional screen homothetic coisotropic submanifold of semi-Riemannian space form $R_{2}^{n+4}(c)$. If both the equality cases of (4.6) and (4.12) hold for all $p \in M$ simultaneously then $c=0$.

\section{Some applications}

In this section, we are going to obtain some corollaries by the help of relations given in the previous section and investigate the notion of ideality for some examples of screen conformal coisotropic lightlike submanifold of a semi-Euclidean space.

From (4.8) inequality in $(c)$ statement of Theorem 4.1 we obtain the following corollary:

Corollary 5.1. Let $(M, g, S(T M))$ be a screen locally conformal coisotropic lightlike submanifold of $(n+4)$-dimensional semi-Euclidean space $R_{2}^{n+4}$ with $\varphi_{1}>0$ and $\varphi_{2}<0$ then we have

$$
2 r_{S(T M)}(p) \leq n^{2}\left\|H^{\prime}(p)\right\|^{2}-2 \sum_{i, j=1}^{n} \varphi_{2}\left(D_{i j}^{2}\right)^{2} .
$$

If the equality case of (5.1) holds for all point $p \in M$, then any $n$-dimensional leaf of $S(T M)$ is ideal.

Now, we give an example of coisotropic lightlike submanifolds as follows:

Example 5.1. Let $\widetilde{M}=\left(R_{2}^{7}, \widetilde{g}\right)$, where $R_{2}^{7}$ is a semi-Euclidean space of signature $(-,-,+,+,+,+,+)$ with respect to the canonical basis

$$
\left\{\partial x_{1}, \partial x_{2}, \partial x_{3}, \partial x_{4}, \partial x_{5}, \partial x_{6}, \partial x_{7}\right\} .
$$

Consider a submanifold $M$ of $R_{2}^{7}$ given by

$$
x_{2}=\left(x_{3}^{2}+x_{5}^{2}\right)^{\frac{1}{2}}, x_{4}=x_{1}, x_{3}>0, x_{5}>0 .
$$


Then we have

$$
\begin{aligned}
S(T M)= & \operatorname{Span}\left\{X_{1}=x_{5} \partial x_{2}+x_{2} \partial x_{5}, X_{2}=\partial x_{6}, X_{3}=\partial x_{7}\right\}, \\
\operatorname{Rad} T_{p} M= & \operatorname{Span}\left\{\xi_{1}=\partial x_{1}+\partial x_{4}, \xi_{2}=x_{2} \partial x_{2}+x_{3} \partial x_{3}+x_{5} \partial x_{5}\right\}, \\
\operatorname{tr}(T M)= & \operatorname{Span}\left\{N_{1}=\frac{1}{2}\left(-\partial x_{1}+\partial x_{4}\right),\right. \\
& \left.N_{2}=\frac{1}{2}\left(-x_{2} \partial x_{2}+\partial x_{3}-x_{5} \partial x_{5}\right)\right\} .
\end{aligned}
$$

By direct computations, we get

$$
\begin{array}{r}
\widetilde{\nabla}_{\xi_{1}} X_{1}=\widetilde{\nabla}_{\xi_{1}} X_{2}=\widetilde{\nabla}_{\xi_{1}} X_{3}=\widetilde{\nabla}_{\xi_{1}} \xi_{2}=0, \\
\widetilde{\nabla}_{X_{1}} X_{1}=x_{2} \partial x_{2}+x_{5} \partial x_{5}, \widetilde{\nabla}_{X_{2}} X_{2}=\widetilde{\nabla}_{X_{3}} X_{3}=0 .
\end{array}
$$

Using the Gauss and Weingarten formulas, we see that both $D^{1}$ and $C^{1}$ vanish on $\Gamma(S(T M))$,

$$
\begin{array}{r}
D^{2}\left(X_{1}, X_{2}\right)=D^{2}\left(X_{1}, X_{3}\right)=D^{2}\left(X_{2}, X_{2}\right)=D^{2}\left(X_{2}, X_{3}\right)=D^{2}\left(X_{3}, X_{3}\right)=0, \\
D^{2}\left(X_{1}, X_{1}\right)=-\left(x_{3}\right)^{2}, C^{2}\left(X_{1}, X_{1}\right)=\frac{1}{2}, C^{2}\left(X_{1}, X_{2}\right)=C^{2}\left(X_{2}, X_{2}\right)=0 .
\end{array}
$$

Consequently, we obtain $M$ is a screen conformal coisotropic lightlike submanifold with $\varphi_{1}$ is arbitrary and $\varphi_{2}=-\frac{1}{2\left(x_{3}\right)^{2}}$.

Consider $M^{\prime}$ to be a 3-dimensional leaf of $S(T M)$. Then it follows that

$$
T M^{\prime}=\operatorname{Span}\left\{X_{1}, X_{2}, X_{3}\right\}
$$

By straightforward computations, it can be seen that the equality case of (5.1) holds. Therefore, $M^{\prime}$ is an ideal leaf.

Similarly, examples for other cases can be given.

Now, we recall the following well known lemma of B.-Y. Chen [2].

Lemma 5.1. If $n>2$ and $a_{1}, \ldots, a_{n}$, a are real numbers such that

$$
\left(\sum_{i=1}^{n} a_{i}\right)^{2}=(n-1)\left(\sum_{i=1}^{n} a_{i}^{2}+a\right),
$$

then

$$
2 a_{1} a_{2} \geq a,
$$

with equality holding if and only if

$$
a_{1}+a_{2}=a_{3}=\cdots=a_{n} .
$$

Using (4.4), Lemma 5.1 and following a smilar proof way of Lemma 3.2 in [2] or Theorem 4.2 in [19], we have the following:

Corollary 5.2. Let $(M, g, S(T M))$ be an $(n+2)$ dimensional $(n>2)$ screen conformal coisotropic lightlike submanifold of a semi-Euclidean space of index 2. Suppose $\varphi_{k}>0$ for $k=1,2$ and $\Pi=\operatorname{Span}\left\{e_{1}, e_{2}\right\}$ is a 2-dimensional nondegenerate plane section of $T_{p} M$. Then we have

$$
r_{S(T M)}(p)-K(\Pi) \leq \frac{1}{8}\left(\frac{n^{2}(n-2)}{n-1}+1\right)\left\|H^{\prime}(p)\right\|^{2} .
$$

If the equality case of (5.2) holds for all $p \in M$ then $S(T M)$ is totally geodesic.

Now, we give another example of screen conformal coisotropic lightlike submanifold as follows: 
Example 5.2. Let $M$ be a submanifold of $R_{2}^{7}$ given by

$$
\begin{array}{r}
x_{1}=u^{1}, x_{2}=u^{2}, x_{3}=u^{1} \sin u^{3}, \\
x_{4}=u^{1} \cos u^{3}, x_{5}=u^{4}, x_{6}=u^{2}, x_{7}=u^{5} .
\end{array}
$$

Then we have

$$
\begin{aligned}
\operatorname{Rad} T M=\operatorname{Span}\left\{\xi_{1}\right. & =\partial x_{1}+\sin u^{3} \partial x_{3}+\cos u^{3} \partial x_{4}, \\
\xi_{2} & \left.=\partial x_{2}+\partial x_{6}\right\} \\
S(T M)=\operatorname{Span}\left\{Z_{1}\right. & =u^{1} \cos u^{3} \partial x_{3}-u^{1} \sin u^{3} \partial x_{4}, Z_{2}=\partial x_{5}, \\
Z_{3} & \left.=\partial x_{7}\right\} \\
\operatorname{tr}(T M)=\operatorname{Span}\left\{N_{1}\right. & \left.=\frac{1}{2}\left(-\partial x_{1}+\sin u^{3} \partial x_{3}+\cos u^{3} \partial x_{4}\right)\right\} \\
N_{2} & \left.=\frac{1}{2}\left(-\partial x_{2}+\partial x_{6}\right)\right\} .
\end{aligned}
$$

By direct calculations, we obtain

$$
\begin{array}{r}
D^{1}\left(Z_{1}, Z_{1}\right)=-u^{1}, D^{1}\left(Z_{1}, Z_{2}\right)=D^{1}\left(Z_{2}, Z_{2}\right)=D^{1}\left(Z_{2}, Z_{2}\right)=0, \\
C^{1}\left(Z_{1}, Z_{1}\right)=-\frac{1}{2} u^{1}, C^{1}\left(Z_{1}, Z_{2}\right)=C^{1}\left(Z_{2}, Z_{2}\right)=0 .
\end{array}
$$

Therefore, $(M, g, S(T M))$ is a screen conformal coisotropic lightlike submanifold of $R_{2}^{7}$ with $\varphi_{1}=\frac{1}{2}$ and $\varphi_{2}$ is arbitrary.

Consider $M^{\prime}$ to be a 3-dimensional leaf of $S(T M)$. Then it follows that

$$
T M^{\prime}=\operatorname{Span}\left\{Z_{1}, Z_{2}, Z_{3}\right\} .
$$

By straightforward computations, it can be seen that the equality case of (5.2) does not hold. Therefore, $M^{\prime}$ isn't an ideal leaf.

\section{Acknowledgments}

The authors are thankful to the referees for their valuable comments towards the improvement of the paper. The first author of this study is supported by $113 \mathrm{~F} 388$ coded project of the Scientific and Technological Research Council of Turkey (TÜBİTAK).

\section{References}

[1] Bejan, C. L., Duggal, K. L., Global lightlike manifolds and harmonicity, Kodai Math. J., 28(2005), 131-145.

[2] Chen, B.-Y., Some pinching and classification theorems for minimal submanifolds, Arch. Math. (Brno), 60(1993), 568-578.

[3] Chen, B-Y., Strings of Riemannian invariants, inequalities, ideal immersions and their applications, in: The Third Pacific Rim Geom. Conf. Internat Press, Cambridge, MA, (1998), 7-60.

[4] Chen, B.-Y., Riemannian DNA, inequalities and their applications, Tamkang J. of Sci. and Eng., 3(2000), no.3, 123-130.

[5] Chen, B.-Y., Some new obstructions to minimal and Lagrangian isometric immersions, Japan. J. Math., 26(2000), 105-127.

[6] Chen, B.-Y., Pseudo-Riemannian geometry, $\delta$-invariants and applications, World Scientific Publishing, Hackensack, NJ, 2011.

[7] Chen, B.-Y., Mihai, I., Isometric immersions of contact Riemannian manifolds in real space forms, Houston J. Math., 31(2005), 743-764

[8] Chen B.-Y., Vrancken, L., CR-submanifolds of complex hyperbolic spaces satisfying a basic equality, Israel J. Math., 110(1999), $341-358$.

[9] Decu, S., Jahanara, B., Petrović-Torgašev, M., Verstraelen, L., On the Chen character of $\delta(2)$-ideal submanifolds, Krag. J. Math., 32(2009), 37-46.

[10] Dillen, F., Petrovic, M., Verstraelen, L., Einstein, conformally flat and semi-symmetric submanifolds satisfying Chen's equality, Israel J. Math., 100(1997), 163-169.

[11] Duggal, K. L., Warped product of lightlike manifolds, Nonlinear Analysis, 47(2001), no.5, 3061-3072

[12] Duggal, K. L., On existence of canonical screens for coisotropic submanifolds, Int. Electron. J. Geom., 1(2008), no.1, 25-32.

[13] Duggal, K. L., Bejancu, A., Lightlike submanifolds of semi-Riemannian manifolds and applications, Math. and Its Appl., Kluwer Academic Publisher, Dordrecht, 1996.

[14] Duggal, K. L., Jin, D. H., Totally umbilical lightlike submanifolds, Kodai Math. J., 26(2003), no.1, 49-68.

[15] Duggal, K. L., On scalar curvature in lightlike geometry, J. of Geo. and Phys., 57(2007), no.2, 473-481. 
[16] Duggal, K. L., Sahin, B., Differential Geometry of Lightlike Submanifolds, Birkhäuser, Basel, 2010.

[17] Fastenakels, J., Ideal tubular hypersurfaces in real space forms, Arch. Math. (Brno), 42(2006), $295-305$.

[18] Gülbahar, M., Kılıç, E., Keleş, S., Chen-like inequalities on lightlike hypersurfaces of a Lorentzian manifold, J. Inequal. Appl., 2013:266, (2013).

[19] Gülbahar, M., Kılıç, E., Keleş, S., Some inequalities on screen homothetic lightlike hypersurfaces of a Lorentzian manifold, Taiwan J. of Math., 17(2013), no.6, 2083-2100.

[20] Hong, S., Matsumoto, K., Tripathi, M. M., Certain basic inequalities for submanifolds of locally conformal Kaehlerian space forms, SUT J. Math., 4(2005), 75-94.

[21] Jin, D. H., Geometry of coisotropic submanifolds, J. Korea Soc. Math. Educ. Ser. B: Pure Appl. Math., 8(2001), no.1, $33-46$.

[22] Kılıç, E., Şahin, S., Karadaḡ, H. B., Güneş, R., Coisotropic submanifolds of a semi-Riemannian manifold, Turk J. Math., $28(2004), 335-352$.

[23] Kupeli, D. N., Singular Semi-Riemannian Geometry, Kluwer Academic, 1996.

[24] Mihai, I., Ideal $C$-totally real submanifolds in Sasakian space forms, Ann. Mat. Pura Appl., 4(2003), no.182, $345-355$.

[25] Mihai, I., Ideal Kaehlerian slant submanifolds in complex space forms, Rocky Mountain J. Math., 35(2005), no.3, $941-952$.

[26] Özgür, C., Arslan, K., On some class of hypersurfaces in $E^{n+1}$ satisfying Chen's equality, Turk J. Math., 26(2002), $283-293$.

[27] Özgür, C., Tripathi, M. M., On submanifolds satisfying Chen's equality in a real space form, The Arab. J. for Sci. and Eng., 33(2008), Number 2A, 320-330.

[28] Özgür, C., B.-Y. Chen inequalities for submanifolds of a Riemannian manifold of quasi-constant curvature, Turk J. Math., 35(2011), no.3, 501-509.

[29] Özgür, C., De, U. C., Chen inequalities for submanifolds of a Riemannian manifold of nearly quasi-constant curvature, Pub. Math. Debrecen, 82(2013), no. 2, 439-450.

[30] Sahin, B., Screen conformal submersions between lightlike manifolds and semi-Riemannian manifolds and their harmonicity, Int. J. Geom. Methods Mod. Phys., 4(2007), no.6, 987-1003.

[31] Sasahara, T., CR-submanifolds in a complex hyperbolic space satisfying an equality of Chen, Tsukuba J. Math., 23(1999), 565-583.

[32] Şentürk, Z., Verstraelen, L., Chen ideal Kaehler hypersurfaces. Taiwan J. Math., 12(2008), no.7, 1597-1608.

[33] Tripathi, M. M., Certain basic inequalities for submanifolds in $(\kappa, \mu)$ space, Recent advances in Riemannian and Lorentzian geometries (Baltimore, MD, 2003), Editors K. L. Duggal and R. Sharma, Amer. Math. Soc., Providence, RI, Contemp. Math., 337(2003), $187-202$.

[34] Tripathi, M. M., Kim, J. S., Kim, S. B., A note on Chen's basic equality for submanifolds in a Sasakian space form, Int. J. Math. Math. Sci., (2003), no. 11, 711-716.

[35] Vilcu, G. E., B.-Y. Chen inequalities for slant submanifolds in quaternionic space forms, Turk J. Math. 34(2010), 115-128.

[36] Vilcu, G. E., On Chen invariants and inequalities in quaternionic geometry, J. of Inequal. Appl., 2013:66, (2013).

[37] Zhang, P., Remarks on Chen's inequalities for submanifolds of a Riemannian manifold of nearly quasi-constant curvature, Vietnam J. of Math., 43(2015), no.3, 557-569.

[38] Zhang, P., Zhang, L., Song, W., Chen's inequalities for submanifolds of a Riemannian manifold of quasi-constant curvature with a semisymmetric metric connection, Taiwan J. Math., 18(2014), no. 6, 1841-1862.

\section{Affiliations}

EROL KILIÇ

ADDRESS: Department of Mathematics, Faculty of Science and Art, İnönü University, 44280, Malatya, TURKEY.

E-MAIL: erol.kilic@inonu.edu.tr

MEHMET GÜLBAHAR

AdDRESS: Department of Mathematics, Faculty of Science and Art, Siirt University, 56100, Siirt, TURKEY. E-MAIL: mehmetgulbahar85@gmail.com 